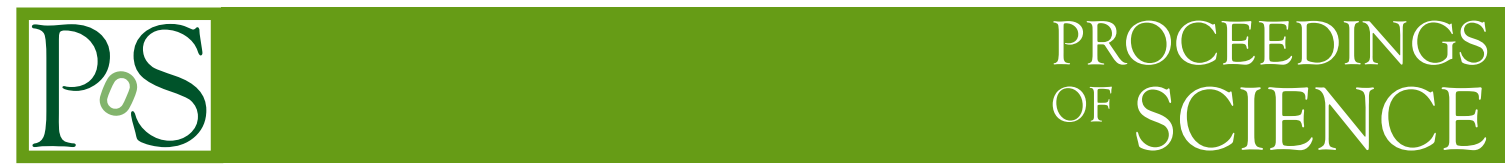

\title{
Semi-inclusive Kaon production at low scales
}

\author{
Juan V. Guerrero* \\ Hampton U. and Jefferson Lab, USA \\ E-mail: juanvg@jlab.org
}

In perturbative QCD, the masses of the hadrons involved in high energy reactions can usually be neglected. In this talk, I will discuss one case in which this may not be a good approximation, namely production of Kaons in electron-proton collisions at low (and not so low) beam energies. In particular, I will present a recent proposal to include hadron masses in theoretical calculations, and how these Hadron Mass Corrections can explain a large discrepancy observed in measurements performed at the HERMES and COMPASS experiments.

XXVI International Workshop on Deep-Inelastic Scattering and Related Subjects (DIS2018) 16-20 April 2018

Kobe, Japan

${ }^{*}$ Speaker. 


\section{Introduction}

There are several ways to access the strange quark PDFs, for instance by analyzing $z$-integrated Kaon multiplicities in Semi-Inclusive Deeply Inelastic Scattering (SIDIS) on deuteron targets. These multiplicities have been measured by the HERMES [1, 2] and COMPASS [3, 4] collaborations showing large discrepancies between their measurements. However, these measurements are sensitive to relatively low values of photon virtualities $(Q \approx 1-4 \mathrm{GeV})$ where the mass, generally denoted by $m$, of the target nucleon and observed hadron, in this case the Kaon $\left(m_{K} \approx 0.5\right.$ $\mathrm{GeV})$, induce non-negligible "Hadron Mass Corrections" (HMCs) of order $\mathscr{O}\left(\mathrm{m}^{2} / Q^{2}\right)[5,6]$.

In this talk, we will show results which quantify these HMCs for Kaon multiplicities in electron deuteron Semi-inclusive Deep Inelastic Scattering (SIDIS) at HERMES and COMPASS. We present evidence that these are not negligible, and may be largely responsible for the apparent discrepancies between the measurements performed by the two collaborations.

\section{Leading order multiplicities at finite $Q^{2}$}

The $z$-integrated hadron multiplicities measured by the HERMES and COMPASS collaborations are defined as a ratio of the semi-inclusive to inclusive cross sections,

$$
M^{h}\left(x_{B}^{\exp }\right)=\frac{\int_{\exp } d x_{B} d Q^{2} \int_{0.2}^{0.8(0.85)} d z_{h} \frac{d \sigma^{h}}{d x_{B} d Q^{2} d z_{h}}}{\int_{\exp } d x_{B} d Q^{2} \frac{d \sigma^{\mathrm{DIS}}}{d x_{B} d Q^{2}}},
$$

where $x_{B}=\frac{Q^{2}}{2 p \cdot q}$ and $Q^{2}=-q^{2}$, namely the Bjorken scaling variable and the virtuality of the exchanged photon respectively, are the usual inclusive invariants, $z_{h}=\frac{p \cdot p_{h}}{p \cdot q}$ is the fragmentation invariant, and the rest of kinematics variables are defined in Fig. 1 left ${ }^{1}$. The integration over the inclusive invariants, $d x_{B} d Q^{2}$, is performed over the bin of nominal value $x_{B}^{\text {exp }}$ with the integration over $d Q^{2}$ being performed within $x_{B}$-dependent limits defined by each experiment's kinematic cuts [3,7]; more details are discussed in Ref. [8]. The $z_{h}$-integration limits are those defined for each experiment, for which we denote COMPASS with a parenthesis.

In collinear factorization, only the light-cone components of momenta enter the cross sections. Therefore, we consider massive scaling variables defined by the relevant light-cone fractions $\xi=$ $q^{+} / p^{+}$, also known as Nachtmann scaling variable, and $\zeta_{h}=p_{h}^{-} / q^{-}$. In the so-called $(p, q)$ frame, where $p$ and $q$ are collinear and have zero transverse momentum, one finds [6]

$$
\begin{aligned}
\xi & \equiv-\frac{q^{+}}{p^{+}}=\frac{2 x_{B}}{1+\sqrt{1+4 x_{B}^{2} M^{2} / Q^{2}}} \\
\zeta_{h} & \equiv \frac{p_{h}^{-}}{q^{-}}=\frac{z_{h}}{2} \frac{\xi}{x_{B}}\left(1+\sqrt{1-\frac{4 x_{B}^{2} M^{2} m_{h}^{2}}{z_{h}^{2} Q^{4}}}\right)
\end{aligned}
$$

\footnotetext{
${ }^{1}$ In fact, COMPASS defines integrated multiplicities as averages over $y$ of the differential ones $\int d z_{h}\left\langle M^{h}\left(x_{b}, y, z_{h}\right)\right\rangle_{y}$ without precisely defining the average symbol; in this talk we will use Eq. (2.1) for both experiments.
} 


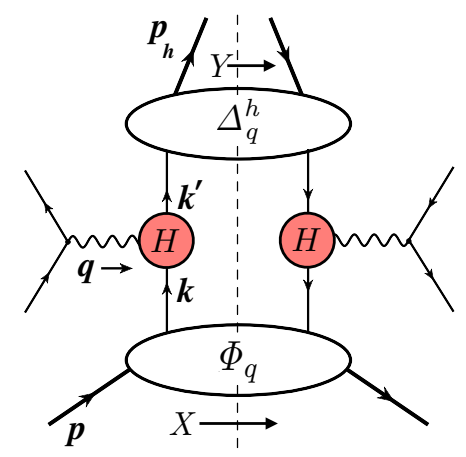

Figure 1: SIDIS handbag diagram and kinematics, where $q$ is the momentum of the photon, $p$ of the target nucleon, $p_{h}$ of the observed hadron, $k$ and $k^{\prime}$ of the partons participating in the hard scattering $H$.

The LO finite- $Q^{2} z$-integrated hadron multiplicity can be written as a factorized expression in terms of quark PDFs, $q$, and FFs, $D_{q}^{h}$, evaluated at the scaling variables $\xi_{h} \equiv \xi\left(1+\frac{m_{h}^{2}}{\zeta_{h} Q^{2}}\right)$ and $\zeta_{h}$ $[8,9]$ :

$$
M^{h}\left(x_{B}^{\mathrm{exp}}\right)=\frac{\sum_{q} e_{q}^{2} \int_{\exp } d x_{B} d Q^{2} \int_{0.2}^{0.8(0.85)} d z_{h} J_{h} q\left(\xi_{h}, Q^{2}\right) D_{q}^{h}\left(\zeta_{h}, Q^{2}\right)}{\sum_{q} e_{q}^{2} \int_{\exp } d x_{B} d Q^{2} q\left(\xi, Q^{2}\right)},
$$

where $J_{h}$ is a Jacobian factor [6]. Note that in the Bjorken limit, Eq. (2.4) reduces to the usual, "massless" $M_{h}^{(0)}$ multiplicity,

$$
M^{h(0)}\left(x_{B}^{\exp }\right)=\frac{\sum_{q} e_{q}^{2} \int_{\exp } d x_{B} d Q^{2} q\left(x_{B}, Q^{2}\right) \int_{0.2}^{0.8(0.85)} d z_{h} D_{q}^{h}\left(z_{h}, Q^{2}\right)}{\sum_{q} e_{q}^{2} \int_{\exp } d x d Q^{2} q\left(x_{B}, Q^{2}\right)} .
$$

\section{Integrated kaon multiplicities}

The HERMES and COMPASS measurements [2, 3, 4] for integrated kaon multiplicities do not appear to be compatible with each other, a well known fact, but discussed mainly focusing on kinematic and binning issues $[7,10,11]$. In this section we discuss how this discrepancy may be in fact apparent and largely due to mass effects. These play an essential role due to the relative low $Q^{2}$ values dominating the HERMES and COMPASS $x_{B}$ bins.

One way to compare HERMES multiplicities to COMPASS multiplicities is by using the ratio between experimental data and theory prediction, because the differences in kinematic cuts and $Q^{2}$ evolution between the two experiments mainly cancel. We calculated and plotted these in Fig. 3 of Ref. [8], using different LO sets of PDFs (MSTW08, CJ15, CT14) [12, 13, 14] and FFs (DSS07, HKNS07) $[15,16]$. There, one can observe a large FF systematic uncertainty, which is due to the poor knowledge we currently have of kaon fragmentation functions. After considering HMCs, the data over theory ratios become flatter, in particular for the COMPASS data.

\subsection{Multiplicities in a massless world}

In order to make a data-to-data comparison of HERMES and COMPASS results, we define "theoretical correction ratios". These make the data from different experimental beam energies directly comparable by producing approximate massless parton multiplicities at a common beam 
energy. They also reduce the theoretical systematic uncertainties (PDFs and FFs choice), and allow one to interpret the corrected multiplicities as parton model multiplicities using Eq. (2.5).

This method consists of two steps. First, we remove the mass effects from the original data multiplying it by the "HMC ratio",

$$
R_{H M C}^{h}=\frac{M^{h(0)}}{M^{h}},
$$

where the numerator is the massless hadron multiplicity, $M^{h(0)}$, defined theoretically by Eq. (2.5) and the denominator is the finite- $Q^{2}$ multiplicity, $M^{h}$, defined by Eq. (2.4).

The second step of this method consists in addressing the difference in the $Q^{2}$ reach of each $x_{B}$ bin of HERMES and COMPASS, often referred as "evolution effects". In this case, we choose to compare the data at COMPASS kinematics. Then, we define an evolution ratio $R_{\text {evo }}^{H \rightarrow C}$ that "brings" HERMES data to COMPASS energy,

$$
R_{\text {evo }}^{H \rightarrow C}=\frac{\left.M^{h(0)}\left(x_{B}^{H E R M E S}\right)\right|_{\text {COMPASS cuts }}}{\left.M^{h(0)}\left(x_{B}^{H E R M E S}\right)\right|_{\text {HERMES cuts }}} .
$$

After removing the mass effects from both sets of data using Eq. (3.1) and multiplying the massless HERMES multiplicity by this evolution ratio, we can define the massless and evolved (at COMPASS $Q^{2}$ ) multiplicities as,

$$
\begin{aligned}
& M_{\text {exp }}^{h(0)} \equiv M_{\text {exp }}^{h} \times R_{H M C}^{h} \quad \text { (for COMPASS) } \\
& M_{\text {exp }}^{h(0)} \equiv M_{\text {exp }}^{h} \times R_{H M C}^{h} \times R_{\text {evo }}^{H \rightarrow C} \quad \text { (for HERMES) }
\end{aligned}
$$

The correction ratios are plotted in Fig. 2, where we find that hadron mass effects are dominant compared to evolution effects. For COMPASS, the corrections are smaller than at HERMES because the $Q^{2}$ accessed at COMPASS is higher bin by bin than at HERMES due to the higher beam energy.

In Fig. 3, we plot the experimental $K^{+}+K^{-}$multiplicity data $M_{\exp }^{K}$ on the left and the "massless" multiplicities $M_{\text {exp }}^{K(0)}$ on the right using Eqs. (3.3a)-(3.3b). An important remark is that corrections are relatively stable with respect to FF and PDF choice, because the related systematic uncertainties are canceled in the correction ratios defined by Eqs. (3.1) and (3.2).The corrected data also shows a show a negative slope in $x_{B}$ that agrees much better with the $(1-x)^{\beta}$ power law behavior of any PDF, including the s-quark. There are still some discrepancy in the $x_{B}$ slopes and shapes of the two experimental measurements. This indicates that additional effects may play a role on top of the HMCs, or that undetected systematic uncertainties are affecting the measurements.

\subsection{Kaon multiplicity ratios}

Another interesting observable which can be studied is the $K^{+} / K^{-}$multiplicity ratio. In this case the experimental systematic uncertainties and evolution effects are expected to largely cancel in the ratio, as well as theoretical uncertainties like next-to-leading order [17] or higher-twist effects. However, for Kaons there still are residual HMCs, although smaller than for the $K^{+}+K^{-}$ sum, due to the difference in fragmentation functions between $K^{+}$and $K^{-}$(see Fig. 6 of Ref. [8]). 


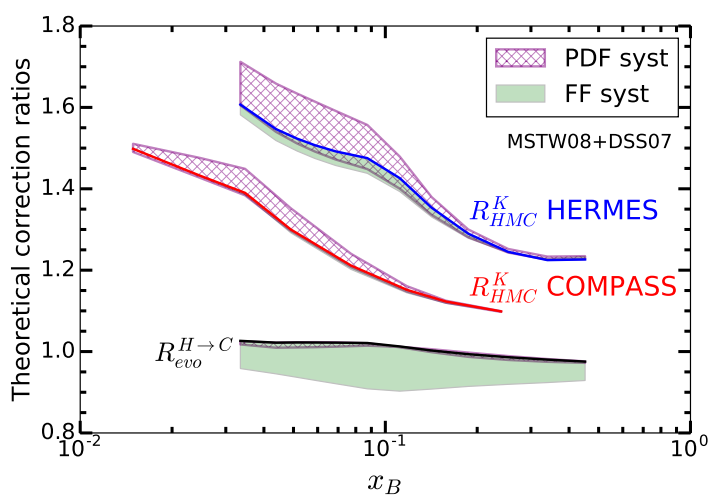

Figure 2: Theoretical correction ratios as a function of $x_{B}$ for charged $K^{+}+K^{-}$multiplicity. The red line correspond to the mass corrections for COMPASS, the blue line correspond to the mass corrections for HERMES while the black line is the HERMES to COMPASS evolution. The green FF systematic uncertainty band for the COMPASS $R_{H M C}^{K}$ is very small compared to the HERMES case and almost invisible in the plot. The purple hashed PDF systematic uncertainty band for $R_{\text {evo }}^{H \rightarrow C}$ is very small compared to the FF systematic uncertainty.
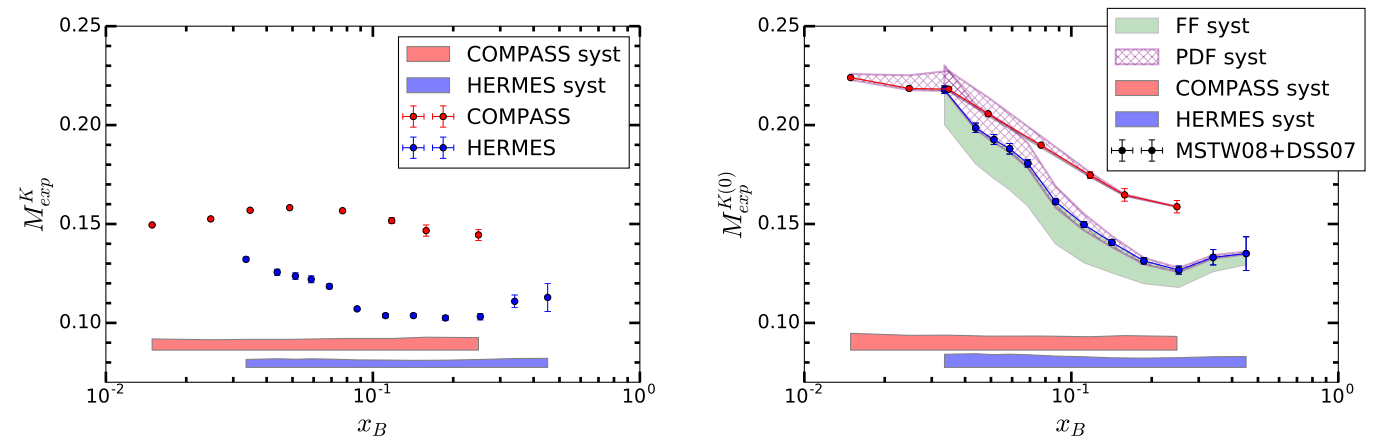

Figure 3: Right: Experimental data for integrated kaon Multiplicities $\left(K^{+}+K^{-}\right)$. Left: Massless multiplicities at a common $Q^{2}$ after applying the theoretical correction ratios given by Eq. (3.3) to the data shown on the right.
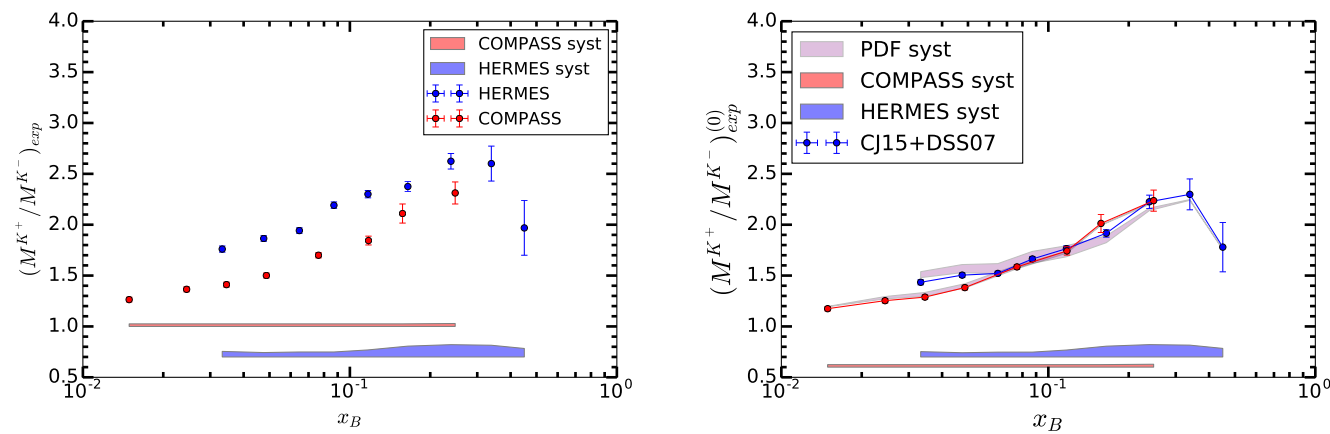

Figure 4: Right: Experimental data for integrated kaon Multiplicities $\left(K^{+} / K^{-}\right)$. Left: Massless multiplicities at a common $Q^{2}$ after applying the theoretical correction ratios given by Eq. (3.3) to the data shown on the right. 
The original and "massless" $K^{+} / K^{-}$data, for both HERMES and COMPASS experiments, are plotted in the left and right panels of Fig. 4. In this case, the slopes are already compatible in the original data but there is a discrepancy in size. After removing the mass effects, the "massless" experimental kaon ratios become fully compatible between the two experiments except maybe last HERMES $x_{B}$ bin, which seems to have a drastic change in slope, as it was the case also for the sum $K^{+}+K^{-}$. Unfortunately, this "hockey stick" shape lies just outside the COMPASS range in $x_{B}$. The origin of this slope change remains to be understood, but it may simple be due to a statistical fluctuation.

\section{Acknowledgments}

We thank C. Van Hulse, A. Bressan, W. Melnitchouk and N. Sato for helpful discussions. This work was supported by the DOE contract No. DE-AC05-06OR23177, under which Jefferson Science Associates, LLC operates Jefferson Lab and DOE contract DE-SC0008791.

\section{References}

[1] A. Airapetian et al. [HERMES Collaboration], Phys. Rev. D 89 (2014) no.9, 097101

[2] A. Airapetian et al. [HERMES Collaboration], Phys. Rev. D 87 (2013) 074029

[3] E. Seder [COMPASS Collaboration], PoS DIS 2015 (2015) 214.

[4] C. Adolph et al. [COMPASS Collaboration], Phys. Lett. B 767 (2017) 133

[5] A. Accardi, T. Hobbs and W. Melnitchouk, JHEP 0911 (2009) 084

[6] J. V. Guerrero, J. J. Ethier, A. Accardi, S. W. Casper and W. Melnitchouk, JHEP 1509 (2015) 169

[7] E. C. Aschenauer et al. [HERMES Collaboration], Phys. Rev. D 92 (2015) no.9, 098102

[8] J. V. Guerrero and A. Accardi, Phys. Rev. D 97 (2018) no.11, 114012

[9] J. V. Guerrero and A. Accardi, PoS QCDEV 2017 (2017) 055, arXiv:1712.04571 [hep-ph].

[10] M. Stolarski, Phys. Rev. D 92 (2015) no.9, 098101

[11] E. Leader, A. V. Sidorov and D. B. Stamenov, Phys. Rev. D 93 (2016) no.7, 074026

[12] A. D. Martin, W. J. Stirling, R. S. Thorne and G. Watt, Eur. Phys. J. C 63 (2009) 189

[13] A. Accardi, L. T. Brady, W. Melnitchouk, J. F. Owens and N. Sato, Phys. Rev. D 93 (2016) no.11, 114017

[14] S. Dulat et al., Phys. Rev. D 93 (2016) no.3, 033006

[15] D. de Florian, R. Sassot and M. Stratmann,

[16] M. Hirai, S. Kumano, T.-H. Nagai and K. Sudoh, Phys. Rev. D 75 (2007) 094009

[17] C. W. Kao, D. J. Yang and W. C. Chang, arXiv:1807.06524 [hep-ph]. 